\title{
Jets and decays of resonances: Two mechanisms responsible for reduction of the elliptic flow at the CERN Large Hadron Collider (LHC), and restoration of constituent quark scaling
}

\author{
G. Eyyubova, ${ }^{1, *}$ L.V. Bravina, ${ }^{1}$ V.L. Korotkikh,${ }^{2}$ I.P. Lokhtin,${ }^{2}$ \\ L.V. Malinina, ${ }^{2}{ }^{\dagger}$ S.V. Petrushanko, ${ }^{2}$ A.M. Snigirev, ${ }^{2}$ and E. Zabrodin ${ }^{1, *}$ \\ 1 Department of Physics, University of Oslo, PB 1048 Blindern, N-0316 Oslo, Norway \\ ${ }^{2}$ Skobeltsyn Institute of Nuclear Physics, Moscow State University, RU-119991 Moscow, Russia
}

(Dated: April 20, 2022)

\begin{abstract}
The formation and evolution of the elliptic flow pattern in $\mathrm{Pb}+\mathrm{Pb}$ collisions at $\sqrt{s}=5.5 A \mathrm{TeV}$ and in $\mathrm{Au}+\mathrm{Au}$ collisions at $\sqrt{s}=200 \mathrm{~A} \mathrm{GeV}$ are analyzed for different hadron species within the framework of the HYDJET ++ Monte Carlo model. The model contains both hydrodynamic state and jets, thus allowing for a study of the interplay between the soft and hard processes. It is found that jets terminate the rise of the elliptic flow with increasing transverse momentum. Since jets are more influential at the Large Hadron Collider (LHC) than at the Relativistic Heavy Ion Collider (RHIC), the elliptic flow at LHC should be weaker than that at RHIC. The influence of resonance decays on particle elliptic flow is also investigated. These final state interactions enhance the low- $p_{T}$ part of the $v_{2}$ of pions and light baryons and work toward the fulfillment of idealized constituent quark scaling.
\end{abstract}

PACS numbers: 25.75.-q, 25.75.Ld, 24.10.Nz, 25.75.Dw

\section{INTRODUCTION}

In noncentral collisions between two nuclei the beam direction and the impact parameter vector define a reaction plane for each event. The observed particle yield versus azimuthal angle with respect to the event-by-event reaction plane might carry information on the early collision dynamics [1, 2]. An initial nuclear overlap region has an "almond" shape at nonzero impact parameter. If the produced matter further interacts and quickly thermalizes, the pressure that is built up within the almondshaped region, develops anisotropic pressure gradients. This pressure pushes against the outside vacuum and the matter expands collectively. The result is an anisotropic azimuthal angle distribution of the detected particles. One can expand this azimuthal angle distribution in Fourier series $[3,4]$. The second coefficient of the expansion $v_{2}$ is called elliptic flow:

$$
v_{2} \equiv\langle\cos 2 \phi\rangle=\left\langle\frac{p_{x}^{2}-p_{y}^{2}}{p_{x}^{2}+p_{y}^{2}}\right\rangle .
$$

Here, $\phi$ is the azimuthal angle of a particle relative to the reaction plane, and $p_{x}$ and $p_{y}$ are the in-plane and out-of-plane components of the transverse momentum of a particle, respectively.

\footnotetext{
*Also at Skobeltsyn Institute of Nuclear Physics, Moscow State University, RU-119991 Moscow, Russia

$\dagger$ Also at Department of Physics, University of Oslo, Post Box 1048 Blindern, N-0316 Oslo, Norway and Joint Institute for Nuclear Researches, Dubna, Moscow Region, RU-141980, Russia
}

It was found $[2,5]$ that anisotropic flow is a self-quenching phenomenon since it reduces spatial anisotropy as it evolves. Therefore, the observed elliptic flow must originate at early stages of the collision when the anisotropy is still present in the system, and no flow is generated when the spherical symmetry is restored. Thus, the elliptic flow can provide information about hot and dense matter created in relativistic heavy-ion collisions.

Although the elliptic flow has been extensively studied both theoretically and experimentally (see, e.g., Refs. [6, 7] and references therein), its behavior in $\mathrm{Pb}$ $+\mathrm{Pb}$ collisions at Large Hadron Collider (LHC) energy $\sqrt{s}=5.5 \mathrm{~A} \mathrm{TeV}$, compared with that at the Relativistic Heavy Ion Collider (RHIC), $\sqrt{s}=200 A \mathrm{GeV}$, remains completely unclear (for a recent review see Ref. [8]). Some models predict further increase of the $v_{2}$ at LHC, while others favor a similarity, $v_{2}^{\mathrm{LHC}} \approx v_{2}^{\mathrm{RHIC}}$, or even a decrease, $v_{2}^{\text {LHC }}<v_{2}^{\text {RHIC }}$. The difference among the predictions of different models comes from the treatment of various processes at partonic and hadronic levels, such as equilibration, features of quark-hadron phase transition, hadronic cascade, equation of state (EOS), and cross section of partonic scattering, as well as from the initial conditions. To the best of our knowledge, the interplay between the ideal hydrodynamic behavior and jets, that is, the influence of the jets on hydrodynamic flow with rising collision energy, has not been elaborated yet. For this purpose we employ the HYDJET ++ model [9] to estimate the azimuthal anisotropy of particles in $\mathrm{Pb}+$ $\mathrm{Pb}$ collisions at $\sqrt{s}=5.5 \mathrm{~A} \mathrm{TeV}$ and compare the obtained results with calculations for $\mathrm{Au}+\mathrm{Au}$ collisions at the top RHIC energy $\sqrt{s}=200 A \mathrm{GeV}$ and also with the experimental data.

The HYDJET ++ model [9] is a superposition of soft 
and hard parts. These parts are independent and their contribution to the total multiplicity production depends on collision energy and centrality and is tuned by model parameters. The hard part of the model is identical to that of the HYDJET model [10] and can account for jet quenching effect and the shadowing effect [11]. The soft part of the HYDJET ++ event represents the "thermal" hadronic state where multiplicities are determined under assumption of thermal equilibrium [12, 13]. Hadrons are produced on the hypersurface represented by a parametrization of relativistic hydrodynamics with given freeze-out conditions. The model is capable of simultaneously reproducing the main features of heavy-ion collisions at RHIC, namely, (i) hadron spectra and ratios, (ii) radial and elliptic flow, (iii) femtoscopic momentum correlations, as well as (iv) high- $p_{T}$ hadron spectra.

As the fireball expands, its temperature and energy density drop. Finally, at the freeze-out stage the system breaks up into hadrons and their resonances. The effect of resonance decays, namely, final state interactions, on the resulting elliptic flow of particles is quite important at both LHC and RHIC energies. Here, we are going to benefit from the rich table of baryon and meson resonance states implemented in HYDJET ++ .

The paper is organized as follows. Features of the model are presented in Sec. II. In Sec. III the influence of jets on the elliptic flow of the most abundant hadrons, such as pions, protons, kaons and lambdas, is studied. We show that the superposition of hydrodynamic flow and jets leads to reduction of the flow at high transverse momenta. Compared with RHIC, the jet fraction at LHC starts to dominate at smaller $p_{T}$, thus effectively decreasing the elliptic flow of all particles. In Section IV we describe the study of the influence of resonance decays on the $v_{2}$ of stable (with respect to strong interaction) particles. At both RHIC and LHC, the effect is found to be the strongest for protons and lambdas, relatively moderate for pions, and almost absent for kaons. While jets modify the (semi)hard part of the $v_{2}\left(p_{T}\right)$ spectra, the contributions from resonance decays alter their soft part. In Sec. V we show that these contributions can account for better realization of the constituent quark scaling of the hadron elliptic flow. Finally, conclusions are drawn in Sec. VI.

\section{THE HYDJET + + GENERATOR}

HYDJET ++ is a Monte Carlo event generator for the simulation of relativistic heavy-ion $A+A$ collisions as the superposition of a soft hydro-type state and a hard multiparton state. Both states are treated independently. HYDJET ++ [9] is a further development of its predecessors: the HYDJET [10] and FASTMC [12, 13] Monte Carlo generators. The soft part is based on a hydrodynamical parametrization of the initial state providing the thermal hadronic state generated on the chemical (single freeze-out scenario) or thermal (thermal freeze- out scenario) freeze-out hypersurfaces represented by a parametrization of relativistic hydrodynamics with given freeze-out conditions $[12,13]$. The mean multiplicity of hadron species crossing the spacelike freeze-out hypersurface is calculated using effective thermal volume approximation. Note that unlike FASTMC in HYDJET++ the value of effective volume of the fireball $V_{\text {eff }}$ is generated for each event separately. $V_{\text {eff }}$ is proportional to the mean number of participating nucleons at the considered centrality (impact parameter $b$ ), which is calculated from the generalization of the Glauber multiple scattering model to the case of independent inelastic nucleon-nucleon collisions. In the case of the thermal freeze-out scenario the system expands hydrodynamically with frozen chemical composition, cools down and finally decays at a thermal freeze-out hypersurface [12]. The two- and three-body decays of the resonances with branching ratios are taken from the SHARE particle decay table [14].

The model for the hard multiparton part of the HYDJET ++ event is the same as that for the HYDJET event generator. A detailed description of the physics framework of this model can be found in Ref. [10]. The approach for multiple scattering of hard partons in dense QCD matter, that is, quark-gluon plasma, is based on an accumulating energy loss, the gluon radiation, and collisional loss being associated with each parton scattering in the expanding quark-gluon fluid.

The routine for generation of a single hard $N N$ collision, PYQUEN $[10,15]$, is constructed as a modification of the jet event obtained with the generator of hadronhadron interactions, PYTHIA_6.4 [16]. The event-byevent simulation procedure in PYQUEN includes the generation of the initial parton spectra with PYTHIA and production vertexes at the given impact parameter, rescattering-by-rescattering simulation of the parton path length in a dense zone, radiative and collisional energy loss, and final hadronization with the Lund string model for hard partons and in-medium emitted gluons. Then, the full hard part of the event includes PYQUEN multijets generated around its mean value according to the binomial distribution. The mean number of jets produced in $A+A$ events is a product of the number of binary $N N$ subcollisions at a given impact parameter and the integral cross section of the hard process in $N N$ collisions with the minimal transverse momentum transfer, $p_{T}^{\min }$. Further details of the model can be found in Refs. $[9,10,12,13]$.

\section{HOW JETS CAN DIMINISH THE ELLIPTIC FLOW AT LHC COMPARED WITH RHIC}

In the following we consider heavy-ion collisions at fixed centrality $\sigma / \sigma_{\text {geo }}=\left[b /\left(2 R_{A}\right)^{2}\right]=42 \%$, corresponding to impact parameter $b \approx 1.3 R_{\mathrm{A}}$. At this centrality the elliptic flow is already quite strong, and the total hadron multiplicity is still high enough to reduce fluctuations in the high- $p_{T}$ region of particle spec- 


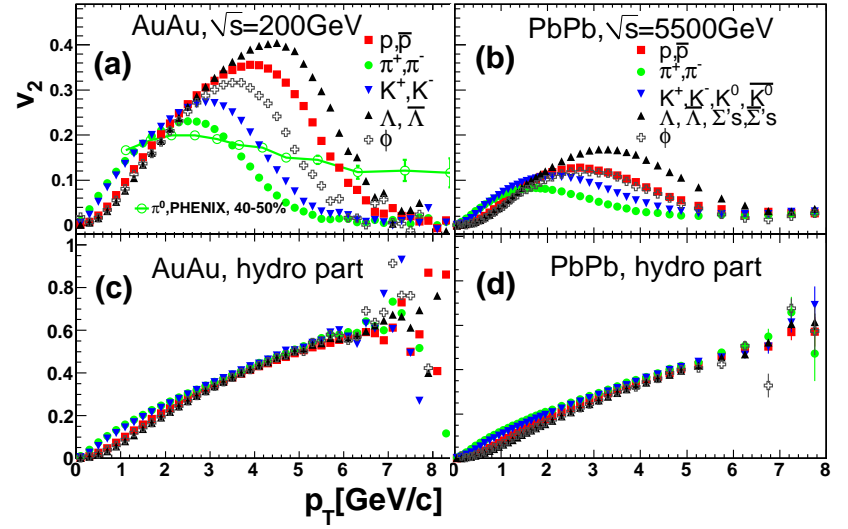

FIG. 1: (Color online) The $p_{T}$ dependence of total elliptic flow (upper row) and its hydro component (bottom row) in the HYDJET ++ model for different hadron species. Left column: $\mathrm{Au}+\mathrm{Au}$ collisions at $\sqrt{s}=200 A \mathrm{GeV}$. Right column: $\mathrm{Pb}+$ $\mathrm{Pb}$ collisions at $\sqrt{s}=5.5 \mathrm{ATeV}$. Centrality for both reactions is fixed at $c=\sigma / \sigma_{\text {geo }}=42 \%$. Data for $\pi^{0}$ flow, shown in (a), are taken from Ref. [18].

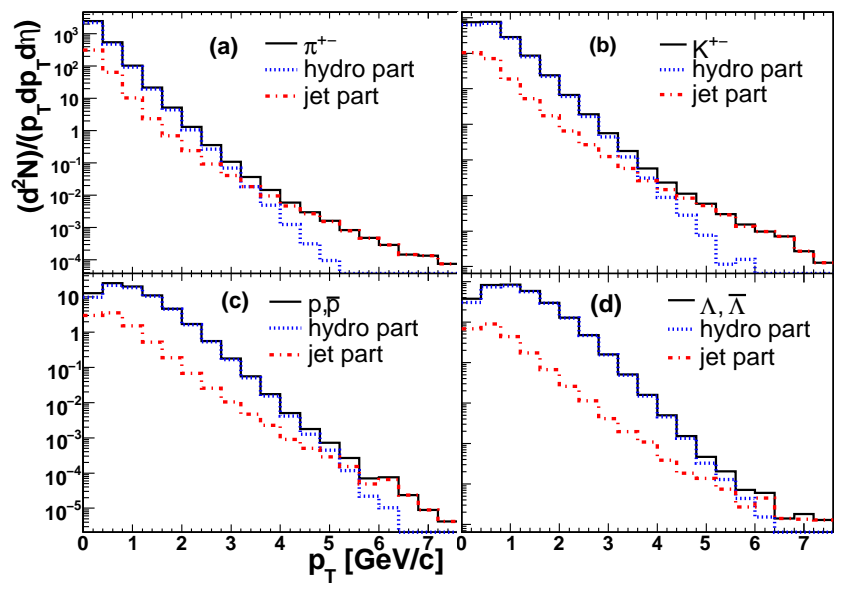

FIG. 2: (Color online) The $p_{T}$ distribution for different hadron species in HYDJET ++ calculations of $\mathrm{Pb}+\mathrm{Pb}$ collisions at $\sqrt{s}=5.5 \mathrm{ATeV}$ with centrality $c=42 \%$.

tra. Figure 1 shows the transverse momentum dependence of elliptic flow coefficient for the most abundant hadron species, namely, pions, kaons, protons, lambdas and sigmas, produced in 1,000,000 gold-gold collisions at $\sqrt{s}=200 A \mathrm{GeV}$ and in ca. 500,000 lead-lead interactions at $\sqrt{s}=5.5 \mathrm{ATeV}$. At least three features should be mentioned here. First, for both reactions, the initial increase of the flow with rising $p_{T}$ is accompanied by the rapid falloff at high values of the transverse momentum. Second, the pronounced feature of the RHIC experimental data [17] reproduced by HYDJET ++ in Fig. 1 is the crossing of baryon and meson branches. Finally, the flow at LHC is almost $50 \%$ lower than the flow at RHIC. We also plot recent data concerning the elliptic flow of neutral pions at RHIC [18] onto the model predictions in Fig. 1(a). The experimental data indicate a slight decrease of the $v_{2}$ at $p_{T} \geq 2 \mathrm{GeV} / c$ and its possible saturation at the $10 \%$ level, whereas in the HYD$\mathrm{JET}++$ calculations the flow in the high- $p_{T}$ domain is much weaker and does not exceed $2-3 \%$. Therefore, the model predictions of the flow excitation functions $v_{2}\left(p_{T}\right)$ above $p_{T} \approx 4 \mathrm{GeV} / c$ should be considered as qualitative ones. However, what is the origin of the flow drop and why is the $v_{2}$ at LHC significantly smaller than the RHIC flow? These peculiarities in the behavior of the elliptic flow can be explained by the interplay between the hydrolike part of the spectra and the jets.

The flow of the hydro part rises monotonically up to $v_{2} \simeq 0.5$ at $p_{T} \simeq 6 \mathrm{GeV} / c$, while the relative contribution of the soft processes to the total particle multiplicity decreases with rising $p_{T}$, so the particles with $p_{T} \gtrsim 6 \mathrm{GeV} / c$ are produced merely via hard processes, that is, jets. The jets themselves indicate some amount of flow due to the jet quenching effect. [The energy loss of the high- $p_{T}$ partons depends on their passing length in the anisotropic matter, thus giving the large yield of the high- $p_{T}$ partons in the short in-plane $(x, z)$ direction compared with that in the long out-of plane $(y, z)$ one.] However, their flow is small and does not exceed $5 \%$ in HYDJET ++ calculations even at full LHC energy. Therefore, the superposition of the hydro part and the jets results in weakening of the elliptic flow after a certain transverse momentum. At LHC, jets turn to dominate over the soft processes at much lower values of $p_{T}$ than at RHIC, thus effectively decreasing the elliptic flow, $v_{2}^{\text {LHC }}<v_{2}^{\text {RHIC }}$ at $p_{T} \geq 3 \mathrm{GeV} / c$. It is worth mentioning that the last result stems from the assumption of similarity of model parameters responsible for the correct description of particle chemical and thermal freeze-out at both energies. (We have considered a naive "scaling" of the existing physical picture of heavy-ion interactions over the two orders of magnitude in center-of-mass energy to the maximum LHC energy $\sqrt{s}=5.5 A \mathrm{TeV}$. The linear extrapolation of the model parameters in $\log (\sqrt{s})$ to the LHC can be found in Ref. [19].) These parameters are listed in Table I. Some of them will probably be modified at LHC more seriously compared with the estimated values. Nevertheless, even if the elliptic flow at LHC in the low- $p_{T}$ region will be stronger than that at RHIC, our condition $v_{2}^{\mathrm{LHC}}<v_{2}^{\mathrm{RHIC}}$ will be accomplished at slightly higher transverse momenta, say, $p_{T} \geq 4 \mathrm{GeV} / c$.

Last but not least, in HYDJET ++ at low $p_{T}$ the elliptic flow is strictly ordered by particle masses. Light particles, such as pions and kaons, have larger flow than heavier ones, such as protons and lambdas. On the other hand, the slope of the $p_{T}$ spectra of light particles is steeper than that of the heavy particles, as can be clearly seen in Fig. 2. As a result, the hydro component of the transverse momentum distribution of heavy hadrons dominates until larger values of $p_{T}$. For instance, for pions and kaons, it determines the spectrum up to $p_{T} \sim 4 \mathrm{GeV} / c$, whereas for protons the hydro part dominates until $p_{T} \sim 5 \mathrm{GeV} / c$. Because of this, the fall of the 
TABLE I: The input parameters for the HYDJET++ generator for the two reactions in question: $T_{\mathrm{ch}}$, temperature at chemical freeze-out; $T_{\mathrm{th}}$, temperature at thermal freeze-out; $\mu_{\mathrm{B}}$, baryon chemical potential; $R_{\mathrm{tran}}^{\max }$, maximum transverse radius at thermal freeze-out; $y_{\text {long }}^{\max }$, maximum longitudinal flow rapidity at thermal freeze-out; $y_{\text {tran }}^{\max }$, maximum transverse flow rapidity at thermal freeze-out; and $p_{T}^{\mathrm{min}}$, minimum transverse momentum of parton-parton scattering. For $\mathrm{Au}+$ $\mathrm{Au}, \sqrt{s}=200 A \mathrm{GeV}$. For $\mathrm{Pb}+\mathrm{Pb}, \sqrt{s}=5.5 A \mathrm{TeV}$.

\begin{tabular}{lcc}
\hline \hline & $\mathrm{Au}+\mathrm{Au}$ & $\mathrm{Pb}+\mathrm{Pb}$ \\
\hline$T_{\mathrm{ch}}$ & $165 \mathrm{MeV}$ & $170 \mathrm{MeV}$ \\
$T_{\mathrm{th}}$ & $100 \mathrm{MeV}$ & $130 \mathrm{MeV}$ \\
$\mu_{\mathrm{B}}$ & $28.5 \mathrm{MeV}$ & 0 \\
$R_{\text {tran }}^{\max }$ & $10 \mathrm{fm}$ & $11 \mathrm{fm}$ \\
$y_{\text {long }}^{\operatorname{man}}$ & 3.3 & 4 \\
$y_{\text {max }}^{\max }$ & 1.1 & 1.1 \\
$p_{T}^{\min }$ & $3.4 \mathrm{GeV} / c$ & $7 \mathrm{GeV} / c$ \\
\hline \hline
\end{tabular}

TABLE II: Yields of the particles produced directly and via the resonance decays at the midrapidity region. Feed-down from weak decays of strange particles is included. For $\mathrm{Pb}+$ $\mathrm{Pb}, c=42 \%$ and $\sqrt{s}=5.5 \mathrm{~A} \mathrm{TeV}$. For $\mathrm{Au}+\mathrm{Au}, c=42 \%$ and $\sqrt{s}=200 A \mathrm{GeV}$.

\begin{tabular}{lccccc}
\hline \hline & $\pi^{ \pm}$ & $K(K)$ & $p(\bar{p})$ & $\Lambda(\Lambda)+\Sigma(\Sigma)$ & $\phi$ \\
\hline $\mathrm{Pb}+\mathrm{Pb}$ & & & & & \\
all & 860 & 185 & 63.8 & 42.3 & 6.55 \\
direct & 169 & 81.4 & 18.6 & 14.2 & 6.5 \\
direct(\%) & 20 & 44 & 30 & 39 & 99 \\
$\mathrm{Au}+\mathrm{Au}$ & & & & & \\
all & 190 & 21.5 & 13.8 & 6.6 & 1.44 \\
direct & 42.9 & 10.4 & 3.2 & 1.2 & 1.43 \\
direct(\%) & 22.5 & 48 & 22 & 18 & 99 \\
\hline \hline
\end{tabular}

elliptic flow with rising transverse momentum occurs for light particles at smaller $p_{T}$, and, at $p_{T} \geq 4 \mathrm{GeV} / c$, the mass ordering of the $v_{2}\left(p_{T}\right)$ spectra is reversed: heaviest particles possess the largest flow.

\section{THE INFLUENCE OF RESONANCE DECAYS}

At RHIC energies the transition to meson-rich matter has been found. As was predicted by Hagedorn [20], at high energies most of the particles will be produced through resonance decays with shifting of the average mass to a heavier sector. Table II shows the contributions of direct and resonant production for various hadron species including feed-down from weak decays for the $\mathrm{Pb}$ $+\mathrm{Pb}$ event sample generated with HYDJET ++ at the top LHC energy. One can see that about $80 \%$ of pions, $70 \%$ of protons, $60 \%$ of $\Sigma$ and $\Lambda$ hyperons and more than $50 \%$ of kaons are produced from the decays of resonances.

The effect of resonance decays should be accounted for

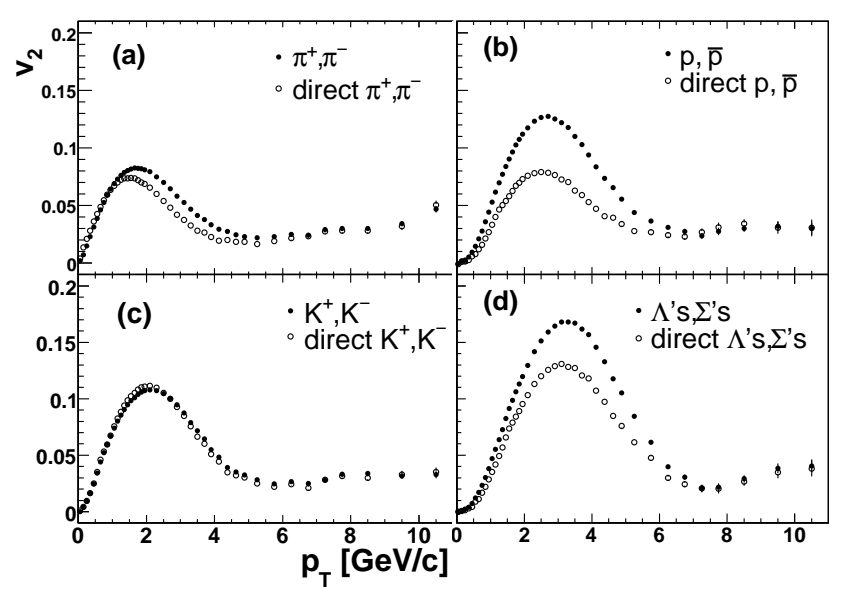

FIG. 3: The $p_{T}$ dependence of elliptic flow of direct hadrons (open symbols) and of all hadrons (full symbols) produced in the HYDJET ++ model for $\mathrm{Pb}+\mathrm{Pb}$ collisions at $\sqrt{s}=$ $5.5 A \mathrm{TeV}$ with centrality $c=42 \%$ : (a) protons, (b) pions, (c) kaons, and (d) lambdas plus sigmas.

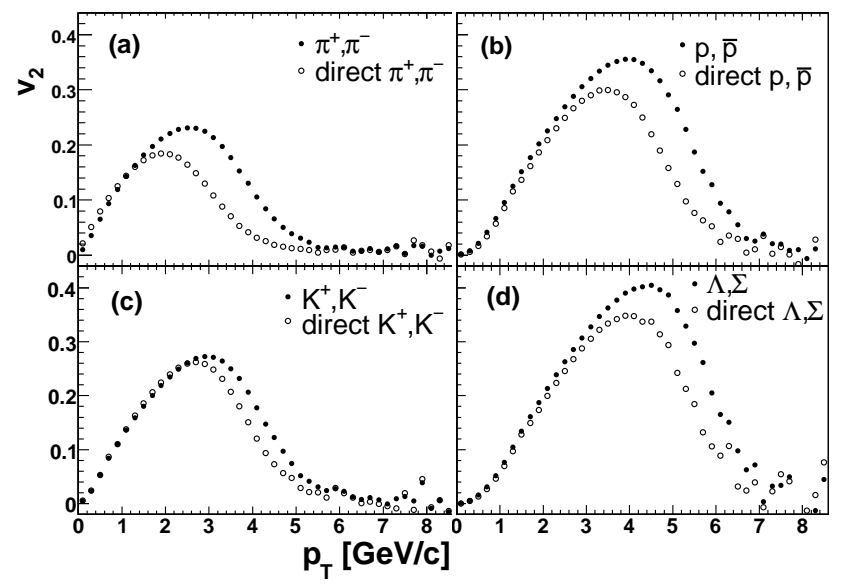

FIG. 4: The same as Fig.3 but for $\mathrm{Au}+\mathrm{Au}$ collisions at $\sqrt{s}=200 A \mathrm{GeV}$.

when one considers the development of the elliptic flow as well. The difference between $v_{2}$ of all these hadrons and $v_{2}$ of only directly produced hadrons is displayed in Figs. 3 and 4 for $\mathrm{Pb}+\mathrm{Pb}$ collisions at LHC and for $\mathrm{Au}+\mathrm{Au}$ collisions at RHIC, respectively. The degree of influence of resonance decays on the strength of elliptic flow is quite different for various hadrons. The effect is strong for protons and $\Sigma+\Lambda$ hyperons, rather moderate for pions, and extremely small for kaons. For all particles, except pions, the resonances either do not alter the flow (kaons) or increase it for both energies in question. Note that in the $p_{T}$ region below $1 \mathrm{GeV} / c$ the elliptic flow of direct pions is larger than the flow of all pions. This finding is in line with the result obtained in relativistic $(2+1)$ hydrodynamics [21]. One can conclude that the soft pions emitted from the decays of resonances have lower momentum anisotropy, whereas heavier particles 


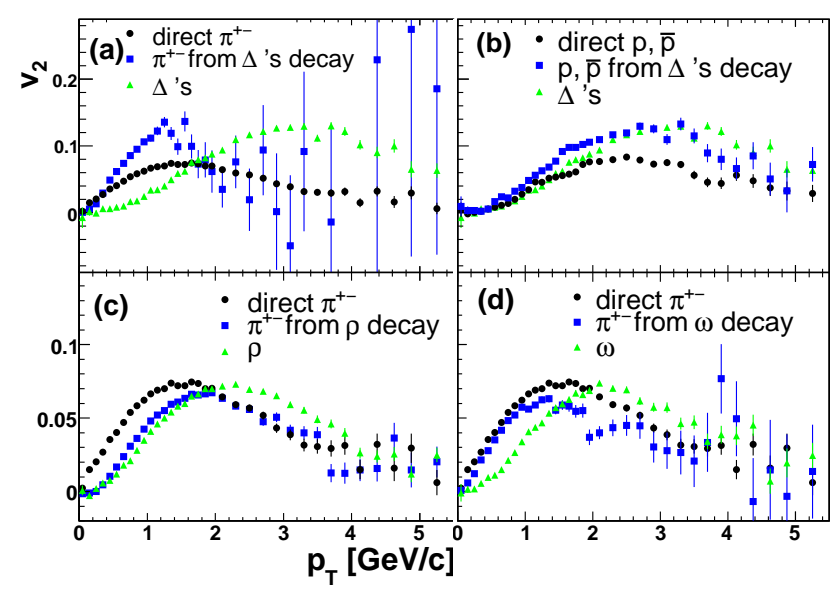

FIG. 5: (Color online) The $p_{T}$ dependence of elliptic flow for (a) charged pions and (b) protons plus antiprotons produced both directly (circles) and in $\Delta$ decays (squares) in the HYDJET ++ model for $\mathrm{Pb}+\mathrm{Pb}$ collisions at $\sqrt{s}=5.5 \mathrm{ATeV}$ with centrality $c=42 \%$. (c) and (d) The same as (a) but for charged pions produced in decays of $\rho$ and $\omega$, respectively. The flow of resonances is shown by triangles.

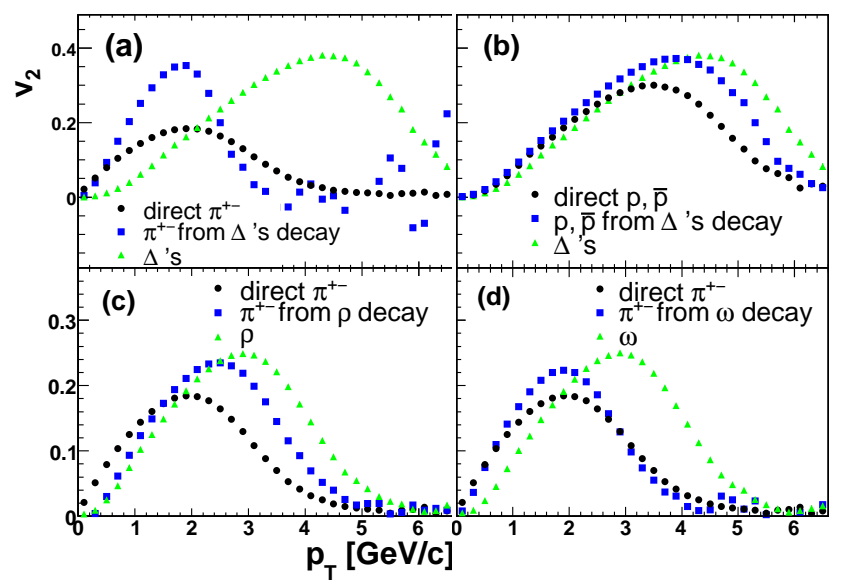

FIG. 6: (Color online) The same as Fig.5 but for $\mathrm{Au}+\mathrm{Au}$ collisions at $\sqrt{s}=200 \mathrm{~A} \mathrm{GeV}$.

and hard pions demonstrate larger elliptic flow of the resonances.

In order to study this peculiarity in detail, let us consider the case of pion and proton flow. Figures 5 and 6 show differences in spectra of secondary pions and protons coming from (anti)delta $\left(\Delta^{++}, \Delta^{+}, \Delta^{0}, \Delta^{-}\right)$decay. When a heavy baryon resonance decays into a secondary baryon plus a pion, the majority of its transverse momentum, because of the decay kinematics, is carried by the baryon, while the pion is produced with lower $p_{T}$. As seen in Figs. 5(a), 5(b), 6(a), and 6(b) the resulting elliptic flows of baryon and pion decay fractions possess similar amplitudes (but not the same $p_{T}$ ) as was carried by the resonance before the decay, that is, $\left\langle v_{2}^{\text {resonance }}\right\rangle \approx\left\langle v_{2}^{\text {baryon }}\right\rangle \approx\left\langle v_{2}^{\text {meson }}\right\rangle$, but $\left\langle p_{T}^{\text {resonance }}\right\rangle \approx$
TABLE III: Relative yields of pions and protons produced directly and via the resonance decays in HYDJET++. Calculations are done for $\mathrm{Au}+\mathrm{Au}$ collisions at RHIC and $\mathrm{Pb}+$ $\mathrm{Pb}$ collisions at LHC with centrality $\mathrm{c}=42 \%$. For $\mathrm{Pb}+\mathrm{Pb}$, $\sqrt{s}=5.5 \mathrm{ATeV}$. For $\mathrm{Au}+\mathrm{Au}, \sqrt{s}=200 \mathrm{~A} \mathrm{GeV}$.

\begin{tabular}{ccccccc}
\hline \hline Hadron & Direct $\rho$ decay & $K^{0}$ & decay & $\omega$ decay & $\Lambda$ decay & $\Delta$ decay \\
\hline $\mathrm{Pb}+\mathrm{Pb}$ & & & & & & \\
$\pi^{ \pm}$ & $22 \%$ & $26 \%$ & $16 \%$ & $11 \%$ & $2.3 \%$ & $1.8 \%$ \\
$p, \bar{p}$ & $30 \%$ & - & - & - & $27 \%$ & $15 \%$ \\
$\mathrm{Au}+\mathrm{Au}$ & & & & & & \\
$\pi^{ \pm}$ & $22.5 \%$ & $21 \%$ & $17.8 \%$ & $8.4 \%$ & $2.3 \%$ & $1.8 \%$ \\
$p, \bar{p}$ & $23 \%$ & - & - & - & $30.4 \%$ & $16 \%$ \\
\hline \hline
\end{tabular}

$\left\langle p_{T}^{\text {baryon }}\right\rangle>\left\langle p_{T}^{\text {meson }}\right\rangle$. Since the transverse momentum spectrum of the produced pions is softer, the pion elliptic flow gets an extra boost at low $p_{T}$ from the intermediate$p_{T}$ flow of heavy resonances see

$$
\text { Figs. 5(a)and6(a) }
$$

. In contrast, the secondary baryon has practically the same $v_{2}\left(p_{T}\right)$ dependence as that of the mother particle, as shown in Figs. 5(b) and 6(b).

The relative contributions of different resonance channels to the yields of protons and pions at RHIC and LHC energies are presented in Table III. We see that, for example, lambdas and deltas alone, together with their antistates, produce about $45 \%$ of all protons and antiprotons. On the other hand, the contribution of baryon resonances to meson spectra is relatively weak. As follows from Table III, many secondary pions are produced from $\rho$ and $\omega$ mesons. The momentum distributions of these pions are quite different, as can be seen in Figs. 5(c), $5(\mathrm{~d}), 6(\mathrm{c})$, and $6(\mathrm{~d})$.

Elliptic flow of pions from the $\rho \rightarrow \pi \pi$ decay almost coincides with $v_{2}^{\rho}$, while in the three-particle decay $\omega \rightarrow \pi \pi \pi$ pions are getting an obviously softer $p_{T}$ distribution, and thus their elliptic flow is transferred to the softer $p_{T}$ region compared with $v_{2}^{\omega}\left(p_{T}\right)$. Although the contributions to the elliptic flow of pions coming from baryon and meson resonances at $p_{T} \leq 1.5 \mathrm{GeV} / c$ effectively compensate each other, the resulting elliptic flow appears to be a bit lower than that of directly produced pions. For higher transverse momenta the contribution from the decays of $K$ mesons and heavy resonances determines the observed excess of the resulting pion flow over the direct pion flow.

In general, the resonance contributions sometimes increase and sometimes decrease the initial elliptic flow assigned to directly produced particles. Some hadrons, such as the $\phi$ meson, do not get a feed-down from the resonances, and their flow profiles remain unchanged. Modification of the elliptic flow of pions and light baryons, especially pronounced at LHC energy, can lead to violation of the hydro-induced mass hierarchy in the $v_{2}\left(p_{T}\right)$ 

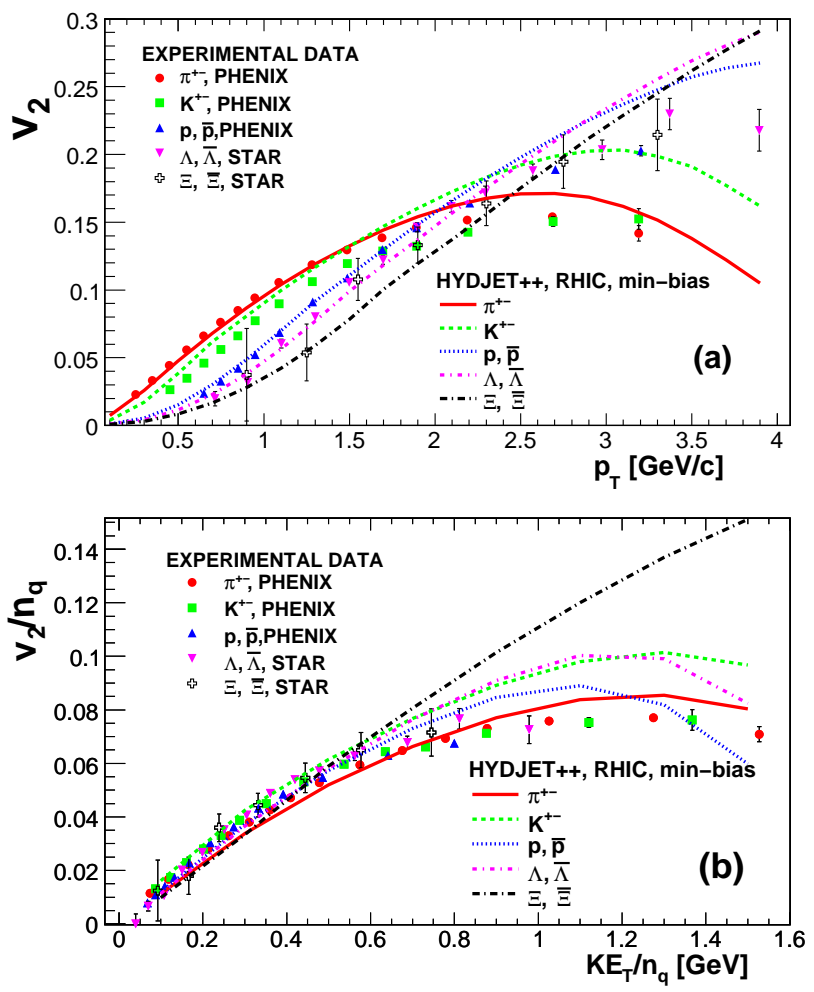

FIG. 7: (Color online) (a) The $p_{T}$ dependence of elliptic flow in the HYDJET ++ model for different hadron species (lines) and comparison with RHIC data (symbols). (b) The same as (a) but for scaling variables, $v_{2} / n_{q}$ vs $K E_{T}$.

sector.

\section{NUMBER-OF-CONSTITUENT-QUARK SCALING}

One of the most interesting features observed in the development of hadron elliptic flow at RHIC is the so-called number-of-constituent-quark (NCQ) scaling [22, 23]. It appears that elliptic flow of any hadron species depends on the transverse kinetic energy $K E_{T} \equiv m_{T}-m_{0}$ in a similar manner provided both $v_{2}$ and $K E_{T}$ are divided by the number of constituent quarks, $n_{q}$, in a given hadron; thas is, $n_{q}=2$ for a meson and $n_{q}=3$ for a baryon. The observance of the NCQ scaling in a broad kinematic range implies the formation of elliptic flow already on a partonic level. Recent experimental studies based on higher statistics indicate that the scaling holds up only until $K E_{T} / n_{q} \approx 1 \mathrm{GeV}[17]$.

The experimental data on particle elliptic flow and NCQ scaling in 1,000,000 minimum bias $\mathrm{Au}+\mathrm{Au}$ collisions at $\sqrt{s}=200 \mathrm{~A} \mathrm{GeV}$ are shown in Fig. 7 together with the results of the HYDJET ++ simulations. Since no information about quark content is used in the hydro part of the HYDJET ++ model, the scaling might not necessarily have been observed. For instance, a $\phi$ meson

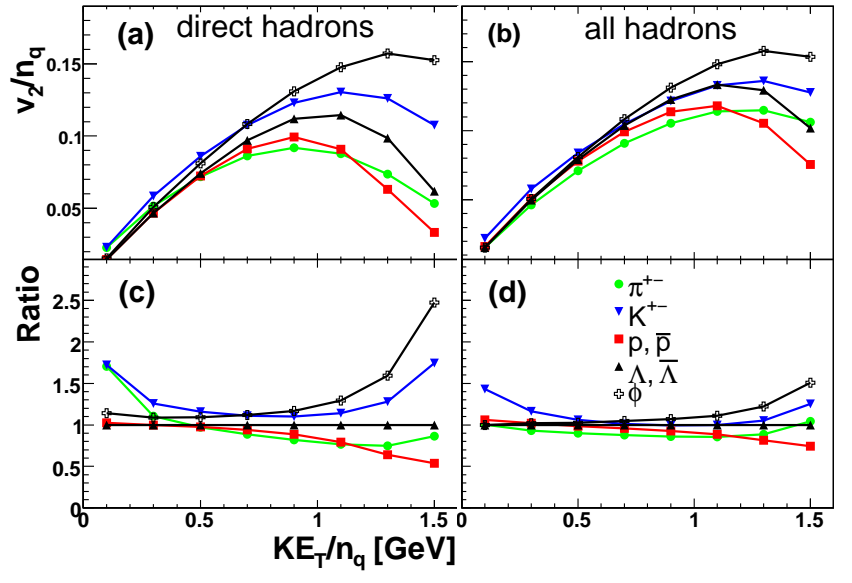

FIG. 8: (Color online) Upper row: The $K E_{T} / n_{q}$ dependence of elliptic flow for (a) direct hadrons and (b) all hadrons produced in the HYDJET ++ model for $\mathrm{Au}+\mathrm{Au}$ collisions at $\sqrt{s}=200 A \mathrm{GeV}$ with centrality $c=42 \%$. Bottom row: The $K E_{T} / n_{q}$ dependence of the ratios $\left(v_{2} / n_{q}\right) /\left(v_{2}^{\Lambda} / 3\right)$ for (c) direct hadrons and (d) all hadrons.

is heavier than a proton and, according to the mass ordering, its normalized flow $v_{2}^{\phi}\left(p_{T}\right) / 2$ is larger than the proton flow $v_{2}^{p}\left(p_{T}\right) / 3$ already at $p_{T} \geq 0.8 \mathrm{GeV} / c$. On the other hand, the elliptic flow in the model is fit to describe experimental data in the low- $p_{T}$ range, as displayed in Fig. $7(\mathrm{a})$. Therefore, the dependence $v_{2} / n_{q}\left(K E_{T} / n_{q}\right)$ may also exhibit the scaling trend. The model calculations presented in Fig. 7(b) seem to obey the approximate NCQ scaling up to $K E_{T} / n_{q} \approx 0.7 \mathrm{GeV}$ or even to higher values if we exclude heavy hyperons, such as $\Xi$ and $\bar{\Xi}$, from our consideration. (There are several experimental and theoretical lines of evidence that multistrange hyperons are frozen earlier; see Ref. [24].) Is this just a coincidence, and what is the role of resonance decays?

To answer these questions we plot HYDJET ++ excitation functions $v_{2} / n_{q}\left(K E_{T} / n_{q}\right)$ for direct hadrons only and for all hadrons produced in gold-gold collisions at top RHIC energy in Figs. 8(a) and 8(b), respectively. In order to see possible deviations from the scaling behavior more distinctly, the ratios $v_{2}^{(i)} / n_{q}: v_{2}^{\Lambda} / 3, i=$ $\pi^{ \pm}, K^{ \pm}, p / \bar{p}, \Lambda / \bar{\Lambda}, \phi$ are presented in Figs. $8(\mathrm{c})$ and $8(\mathrm{~d})$. One may note that, for direct hadrons, the NCQ scaling is fulfilled within the $20 \%$ accuracy limit in the interval $0.2 \leq K E_{T} \leq 0.8 \mathrm{GeV}$. At higher and lower transverse energies the curves diverge, as shown in Figs. 8(a) and $8(\mathrm{c})$. The situation is markedly improved after the resonance decays are taken into account, as demonstrated in Figs. 8(b) and 8(d). All ratios, except for kaons at $K E_{T} \leq 0.2 \mathrm{GeV}$, are much closer to unity, and the scaling appears to hold at $10 \%$ up to $K E_{T} \geq 1.0 \mathrm{GeV}$. The spectrum and, therefore, the elliptic flow of $\phi$ mesons remain unchanged because of the absence of resonance feed-down. In contrast, protons and lambdas are significantly boosted by heavy resonances, and their flows are enhanced at $K E_{T} \geq 0.5 \mathrm{GeV}$. 


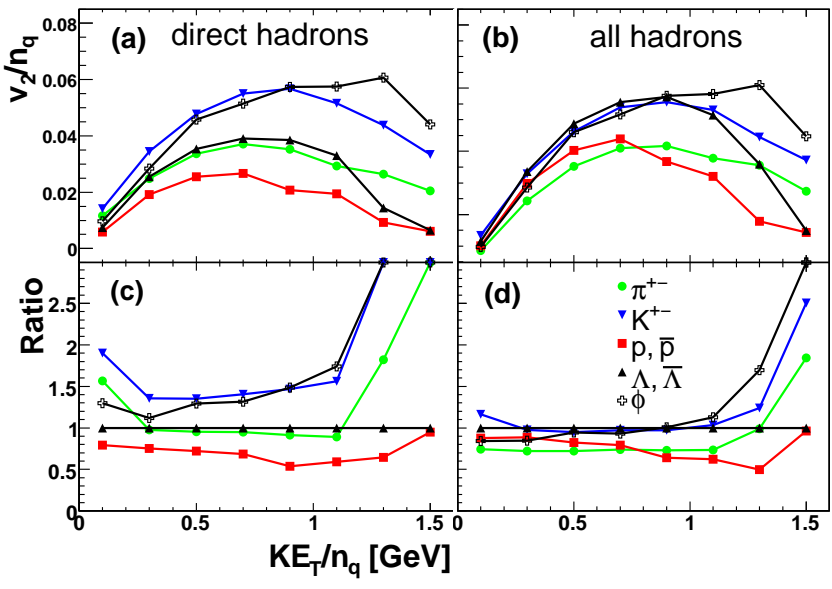

FIG. 9: (Color online) The same as Fig.8 but for $\mathrm{Pb}+\mathrm{Pb}$ collisions at $\sqrt{s}=5.5 \mathrm{~A} \mathrm{TeV}$.

The same mechanism also works at LHC energies. However, because of the influence of jets at relatively low transverse momenta, there is no resemblance of the NCQ scaling for directly produced hadrons, as shown in Figs. 9(a) and 9(c). Here, all curves seem to diverge in the entire transverse energy range. Again, the decays of resonances essentially raise up the elliptic flow of protons and lambdas displayed in Figs. 9(b) and 9(d). However, the effect of jets at LHC is too strong and, although all ratios of particle flows presented in Fig. 9(d) are almost parallel within the energy range $0.1 \leq K E_{T} \leq 1 \mathrm{GeV}$, the realization of theapproximate number-of-constituent-quark scaling becomes worse compared with the RHIC case.

A few remarks on results obtained in other calculations are in order here. The effect of resonance decays on the elliptic flow of stable hadrons in $\mathrm{Au}+\mathrm{Au}$ collisions at RHIC was studied in Ref. [25] in the framework of the coalescence model and in Ref. [26] within the parametrized thermal model. In contrast to our study, the authors of both works postulate that the elliptic flows of directly produced stable particles and resonances obey the number-of-constituent-quark scaling. After that, the resonances account for a substantial part of deviation of pion elliptic flow from the NCQ scaling. We do not assume the automatic performance of the NCQ scaling, but in our model calculations the resonance decays also modify the pion elliptic flow by enhancing it in the intermediate$p_{T}$ region. In accordance with Ref. [25] kaon elliptic flow in our calculations is not affected by the decays of resonances, but we see the strong contribution from heavier resonances to the momentum anisotropies of protons and lambdas. This outlines the importance of a complete table of resonances in any microscopic or macroscopic model designed for the description of heavy-ion collisions. For instance, the present version of the HYDJET ++ model employs ca. 350 baryon and meson states as well as their antistates.

It was suggested recently [27] that NCQ scaling could be used as a unique probe of strongly interacting partonic matter. The scaling holds if the hadrons are produced predominantly from the coalescence of quarks, whereas it is broken if the hadronization proceeds via the string fragmentation. We see, however, that the jet effects become increasingly important at ultrarelativistic energies. Particularly, the interplay between jets and hydrolike flow can cause breaking of the NCQ scaling for the particle elliptic flow at LHC.

\section{CONCLUSIONS}

The elliptic flow pattern in $\mathrm{Pb}+\mathrm{Pb}$ collisions at $\sqrt{s}=$ $5.5 A \mathrm{TeV}$ and in $\mathrm{Au}+\mathrm{Au}$ collisions at $\sqrt{s}=200 A \mathrm{GeV}$ is analyzed for different hadron species in the framework of the HYDJET++ Monte Carlo model. The model contains both parametrized hydrodynamics and jets. This allows one to study the interplay between hard and soft processes and to reveal their role in the formation and evolution of the elliptic flow. It is worth mentioning that the model parameters for the soft hydro component are fixed at RHIC energy to simultaneously describe hadron yields, energy spectra, anisotropic flow, and femtoscopic momentum correlations. These parameters are not expected to change significantly at the LHC energy.

Three general tendencies have been observed. First, at a certain transverse momentum, particles produced via jets start to dominate over the hydrolike particles. Since the flow of jet particles determined merely by the jet quenching is weak, the resulting flow $v_{2}\left(p_{T}\right)$ experiences falloff in the intermediate $p_{T}$ range. Abundant production of jets at LHC will effectively decrease the hadron elliptic flow at smaller values of $p_{T}$ compared with RHIC. Therefore, we expect that the elliptic flow of stable hadrons at LHC will be smaller than its RHIC counterpart, $v_{2}^{\mathrm{LHC}}\left(p_{T}\right)<v_{2}^{\mathrm{RHIC}}\left(p_{T}\right)$, at $p_{T} \gtrsim 3 \mathrm{GeV} / c$.

Second, jets also account for changing of the mass ordering of the hadron elliptic flow at intermediate and high transverse momenta. After the falloff the mass ordering of the $v_{2}\left(p_{T}\right)$ spectra becomes opposite to the initial one; that is, the heavier particle has the larger flow.

Third, resonances are shown to significantly modify the elliptic flows of directly produced hadrons. The $v_{2}\left(p_{T}\right)$ of kaons is not affected by the decays, while the flows of pions and especially protons and lambdas are enhanced. It is the effect of resonance decays that pushes the particle spectra toward the fulfillment of the constituent quark number scaling. At the LHC energy the jets should alter the hadronic elliptic flow already at intermediate transverse momenta, thus completely removing resemblance of the NCQ scaling for directly produced hadrons. After the resonance decays the elliptic flows of different hadrons move closer to each other; however, only the approximate $K E_{T} / n_{q}$ scaling holds. 


\section{Acknowledgments}

Fruitful discussions with L. Sarycheva, J. Schukraft, J. Stachel, and R. Snellings are gratefully acknowledged. We would like to thank I. Arsene, A. Gribushin, and K. Tywoniuk for effective collaboration at different stages of the HYDJET ++ development. This work was sup- ported in part by the QUOTA Program, Norwegian Research Council (NFR), under Contract No. 185664/V30, the Russian Foundation for Basic Research (Grant Nos. 08-02-91001 and 08-02-92496), Grants Nos. 107.2008.2 and 1456.2008.2 of the President of the Russian Federation, and the Dynasty Foundation.
[1] J.-Y. Ollitrault, Phys. Rev. D 46, 229 (1992).

[2] H. Sorge, Phys. Rev. Lett. 82, 2048 (1999).

[3] S. A. Voloshin and Y. Zhang, Z. Phys. C70, 665 (1996).

[4] A. M. Poskanzer and S. A. Voloshin, Phys. Rev. C 58, 1671 (1998).

[5] P. F. Kolb and U. W. Heinz, in Quark Gluon Plasma 3, edited by R. Hwa and X.-N. Wang (World Scientific, Singapore, 2003), p.634.

[6] S. A. Voloshin, A. M. Poskanzer, and R. Snellings, arXiv:0809.2949 [nucl-ex].

[7] P. Sorensen, arXiv:0905.0174 [nucl-ex].

[8] N. Armesto, arXiv:0903.1330 [hep-ph].

[9] I. P. Lokhtin, L. V. Malinina, S. V. Petrushanko, A. M. Snigirev, I. Arsene, and K. Tywoniuk, Comput. Phys. Commun. 180, 779 (2009).

[10] I. P. Lokhtin and A. M. Snigirev, Eur. Phys. J. C46, 211 (2006); http://cern.ch/lokhtin/hydro/hydjet.html .

[11] K. Tywoniuk, I. C. Arsene, L. Bravina, A. B. Kaidalov, and E. Zabrodin, Phys. Lett. B657, 170 (2007).

[12] N. S. Amelin, R. Lednicky, T. A. Pocheptsov, I. P. Lokhtin, L. V. Malinina, A. M. Snigirev, Iu. A. Karpenko, and Yu. M. Sinyukov, Phys. Rev. C 74, 064901 (2006).

[13] N. S. Amelin, R. Lednicky, I. P. Lokhtin, L. V. Malinina, A. M. Snigirev, Iu. A. Karpenko, Yu. M. Sinyukov, I. Arsene, and L. Bravina, Phys. Rev. C 77, 014903 (2008).

[14] G. Torrieri, S. Steinke, W. Broniowski, W. Florkowski,
J. Letessier, and J. Rafelski, Comput. Phys. Commun. 167, 229 (2005).

[15] http://cern.ch/lokhtin/pyquen .

[16] T. Sjostrand, S. Mrenna, and P. Skands, J. High Energy Phys. 0605, 026 (2006); http://home.thep.lu.se/ torbjorn/Pythia.html.

[17] A. Adare et al. (PHENIX Collaboration), Phys. Rev. Lett. 98, 162301 (2007).

[18] R. Wei et al. (PHENIX Collaboration), arXiv:0907.0024 [nucl-ex].

[19] N. Armesto (ed.) et al., J. Phys. G 35, 054001 (2008).

[20] R. Hagedorn, Nuovo Cim. Suppl. 3, 147 (1965).

[21] G. Kestin and U. Heinz, Eur. Phys. J C61, 545 (2009).

[22] J. Adams et al. (STAR Collaboration), Phys. Rev. Lett. 92, 052302 (2004).

[23] S. S. Adler et al. (PHENIX Collaboration), Phys. Rev. Lett. 91, 182301 (2003).

[24] R. Witt, J. Phys. G 34, S921 (2007); P. Chaloupka, M. Sumbera, and L. V. Malinina, Acta Phys. Polon. B40, 1185 (2009).

[25] V. Greco and C. M. Ko, Phys. Rev. C 70, 024901 (2004).

[26] X. Dong, S. Esumi, P. Sorensen, and N. Xu, Phys. Lett. B597, 328 (2004).

[27] J. Tian, J. H. Chen, Y. G. Ma, X. Z. Cai, F. Jin, G. L. Ma, S. Zhang, and C. Zhong, Phys. Rev. C 79, 067901 (2009). 